\title{
Differences between synthetic $\beta$-haematin and native hemozoin crystals
}

\author{
P.A. Carvalho*, L. Coelho**, R.C. Martins*** and F. Nogueira** \\ *ICEMS, Instituto Superior Técnico, Universidade de Lisboa, Av. Rovisco Pais, 1049-001 Lisboa, Portugal \\ **CMDT, UEI de Parasitologia Médica, Instituto de Higiene e Medicina Tropical, Universidade Nova de Lisboa, Rua \\ da Junqueira, nº100, 1349-008 Lisboa, Portugal \\ ***IT, Departamento de Bioengenharia, Instituto Superior Técnico, Universidade de Lisboa, Av. Rovisco Pais, 1049- \\ 001 Lisboa, Portugal
}

Plasmodium falciparum causes the most severe/lethal form of malaria, a parasitic infection that affects 500 million people worldwide and leads to the death of nearly one million/year, about $91 \%$ being due to $P$. falciparum [1]. The malaria pigment or hemozoin crystals (HZ) are formed in an enzyme-independent polymerization of heme released during haemoglobin digestion by the parasite during the intra-erythrocytic life cycle [2]. The quinoline and artemisinin based antimalarials (the only available treatment options) appear to act by disrupting the formation of $\mathrm{HZ}$. Therefore, the development of antimalarial agents based on the physicochemical process of heme crystallization appears a good option to drug design.

We have produced $\beta$-haematin following an assay adapted from the method described by Basilico et al, [3] with modifications. Briefly, hemin (ferriprotoporphyrin IX chloride; Sigma-Aldrich) was dissolved $\mathrm{NaOH}$ and polymerized by the addition of acetic acid and incubation at $37^{\circ} \mathrm{C}$. Resulting pellet washed with dimethyl sulfoxide (DMSO) to remove unpolymerized hemin and resuspended in $\mathrm{H}_{2} \mathrm{O}$. To extract native $\mathrm{HZ}$ (nHZ) from P. falciparum (clone Dd2)-infected erythrocytes, when parasitemia reached $>10 \%$, parasites were harvested by saponin lysis, washed with phosphate-buffered saline (PBS) and sonicated in $2 \%$ sodium dodecyl sulfate (SDS), further washed in $2 \%$ SDS and pellet was resuspended (10 mM Tris- $\mathrm{HCl}(\mathrm{pH} 8.0) ; 0.5 \% \mathrm{SDS} ; 2 \mathrm{mg} / \mathrm{ml}$ of proteinase $\mathrm{K}$ ) and incubated at $37^{\circ} \mathrm{C}$ overnight. The $\mathrm{nHZ}$ pellet was washed in $2 \% \mathrm{SDS}$ and in distilled $\mathrm{H}_{2} \mathrm{O}$, then resuspended in distilled $\mathrm{H}_{2} \mathrm{O}$ water and sonicated again prior to use to minimize aggregation and maintain the $\mathrm{nHZ}$ in suspension. $\beta$-haematin and native $\mathrm{nHZ}$ crystals were analysed by Scanning Electron Microscopy (JEOL 7001FSEM) (Fig.1; A, B.1 and C.1) and Transmission Electron Microscopy (Hitachi H8100) (Fig.1; B.2 and C.2).

Here we report the crystal morphology of $\mathrm{nHZ}$ produced by the Plasmodium falciparum clone Dd2 and of $\beta$-haematin produced with the above protocol. Chemically, HZ is a stable dimer of iron(III)(protoporphyrin[PP]-IX), also known as $\beta$-hematin and consists of heme units dimerized through reciprocal iron-carboxylate bonds [4]. Even though the parasite $\mathrm{nHZ}$ and synthetic $\beta$-hematin are isostructural [4], there are large differences in the crystal morphologies of $\mathrm{nHZ}$ (Dd2) and $\beta$-hematin as evidenced by SEM and TEM images (Fig.1). $\mathrm{nHZ}$ crystals are remarkably uniform in size and shape, adopting a parallelipede morphology with high aspect ratio of about 800x200x200 nm (Fig. $1 \mathrm{C} 1$ and C2). Different protocols to prepare $\beta$-haematin yield diverse material, which can be poorly crystalline and heterogeneous within the same sample [5]. The present results show that $\beta$-haematin is rather homogeneous and constituted by needle-like particles of sizes similar to those presented by $\mathrm{nHZ}$ ones (compare B1 with C1). Higher resolution observations evidence differences between the $\beta$-haematin and $\mathrm{nHZ}$ materials, since the latter seems constituted by uniform flat layers and the former shows flaked ones (Fig. B2).

Morphology seems to be of biological relevance, since structure-function studies revealed that the size and shape of the synthetic crystals influence their ability to activate, for instance, the inflammatory responses in vitro and in vivo [6]. Several protocols have been developed to measure drug inhibition of heme crystallization in vitro [6] although it has been difficult to standardize these assays. In the present study, we report a new method for $\beta$-haematin preparation that renders homogenous crystals, both in shape and size, favourable for reproducible drug inhibition of heme crystallization assays. 
References

1. World Malaria Report, At http://www.who.int/entity/malaria/, 2011.

2. Rosenthal P.J. et al., Mol. Biochem. Parasitol., 83:131-9, 1996.

3. Basilico N. et al., Antimicrob. Chemother., 42:55-60, 1998.

4. Pagola S. et al., Nature., 404:307-10 2000.

5. Slater A.F. et al., Proc. Natl. Acad. Sci. U S A., 88:325-9, 1991.

6. Jaramillo M. et al., PloS one, 4:e6957-e6957, 2009.

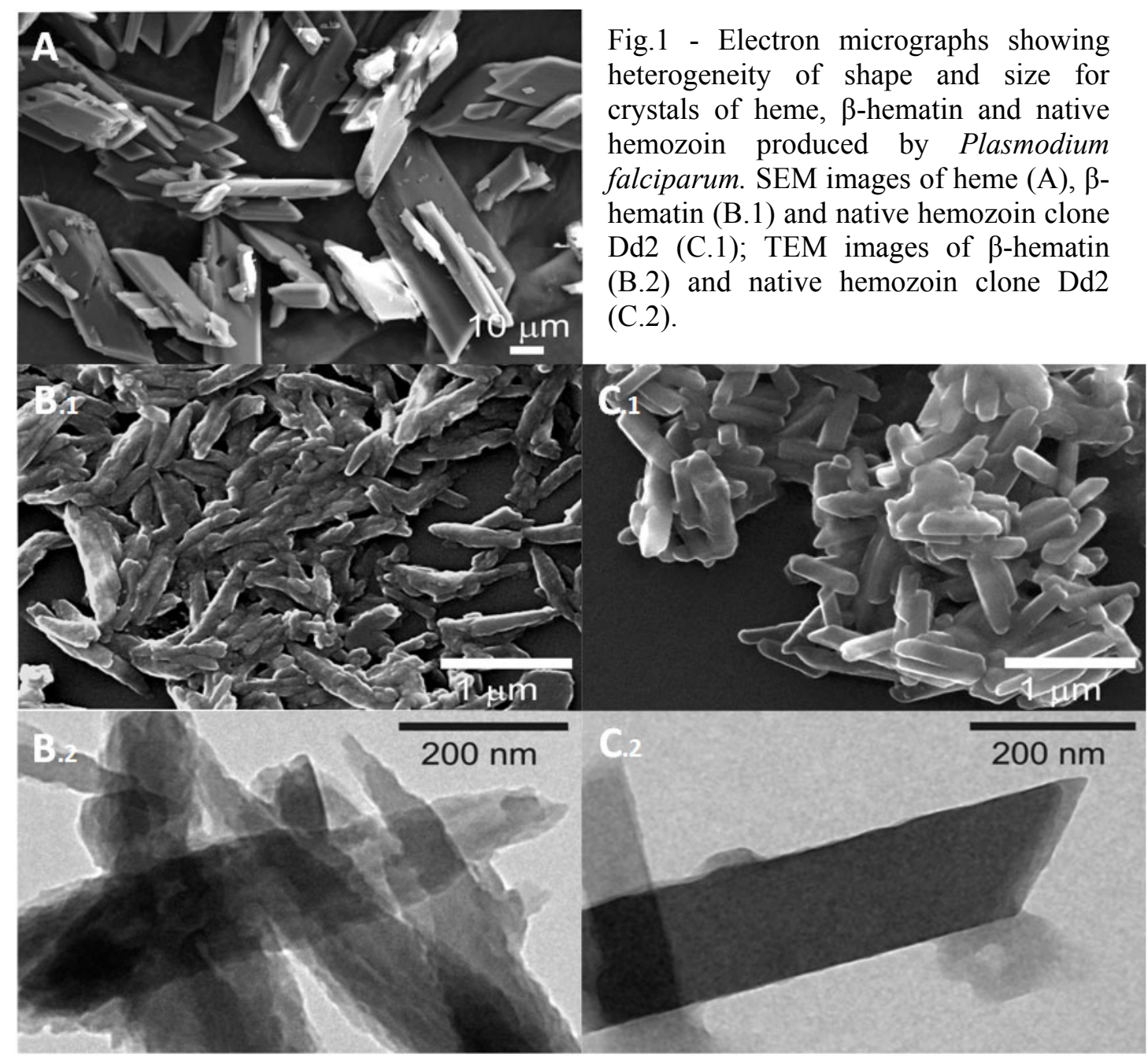

This work was supported by CMDT through PEst-OE/SAU/LA0018/2011 grant and FCT through PTDC/SAUFAR/114864/2009 and PEst-OE/CTM-UI0084/2011 grants. 